\title{
Technique of the research of characteristics of ignition of the condensed systems to variables heat flux
}

\author{
Nikolay N. Zolotorev ${ }^{1, *}$, Vladimir A. Arkhipov ${ }^{1}$, and Eugene A. Maslov ${ }^{2}$ \\ ${ }^{1}$ National Research Tomsk State University, Research Institute of Applied Mathematics and \\ Mechanics, 634050 Tomsk, Russia \\ ${ }^{2}$ National Research Tomsk Polytechnic University, 634050 Tomsk, Russia
}

\begin{abstract}
This paper presents a method for determining the ignition delay time of condensed systems when exposed to radiant heat flux on the sample surface. The heat flow through the focusing lens with the ability to move along the axis was applied to the sample. Changing the geometric characteristics of the optical system can be used for measurements of the ignition of samples condensed systems in a wide range of values.
\end{abstract}

\section{Introduction}

One of the main characteristics in the study of the ignition of condensed systems (CS) is ignition delay time. The ignition delay time depends on the level of heat flux, chemical composition of components, the size of samples, the beam diameter of the emitter, etc.

The ignition delay time play an important role in assessment the explosion of highenergy materials, and also by development of systems of their initiation (igniters, detonators, etc.).

In work [1] the method of measurement of ignition delay time of CS is considered by the given constant radiant heat flux of xenon lamp at which the ignition delay determined by the time interval between the opening of the optical shutter and the moment of emergence of flame on sample surface registered by the ionization sensor and the photo diode. A known method of measuring the ignition delay time of the CS by the radiation of a $\mathrm{CO}_{2}$-laser focused on the sample surface by a lens made of sodium chloride [2]. These techniques allow to take measurements of ignition delay time only at influence of heat flux with the given constant intensity.

As the results of experimental and theoretical studies [3, 4], the ignition characteristics CS essentially depend on the dynamics regime of changes in the heat flux density in the process of initiating. Regularities of ignition of the CS of a heat flux with decreasing or increasing intensity are of interest not only in respect of further development of the thermal theory of ignition, but also are important in practical applications. In actual practice heat exchange at ignition is non-stationary that is carried out in the dynamic regime.

* Corresponding author: nikzolotorev@mail.ru 
In the work [5] presented the technique of research of process of ignition of CS is considered under dynamic conditions of supply of radiant energy. As radiation source in this technique the tubular gas-discharge xenon lamp is used. When opening shutter density of radiant heat flux is changed due to change of current intensity in lamp feed circuit with the special thyristor block.

\section{Method of measurement}

In the work the technique of measurement of ignition delay time of CS which consists in irradiation of surface of sample radiant heat flux through the collecting lens. The collecting lens is moved at a predetermined speed relative to the sample in the measurement process [6]. Before sample collecting lens is installed with the possibility of longitudinal displacement. On the irradiated surface of sample placed a diaphragm with a hole whose center is located on the optical axis of lens (Fig. 1).

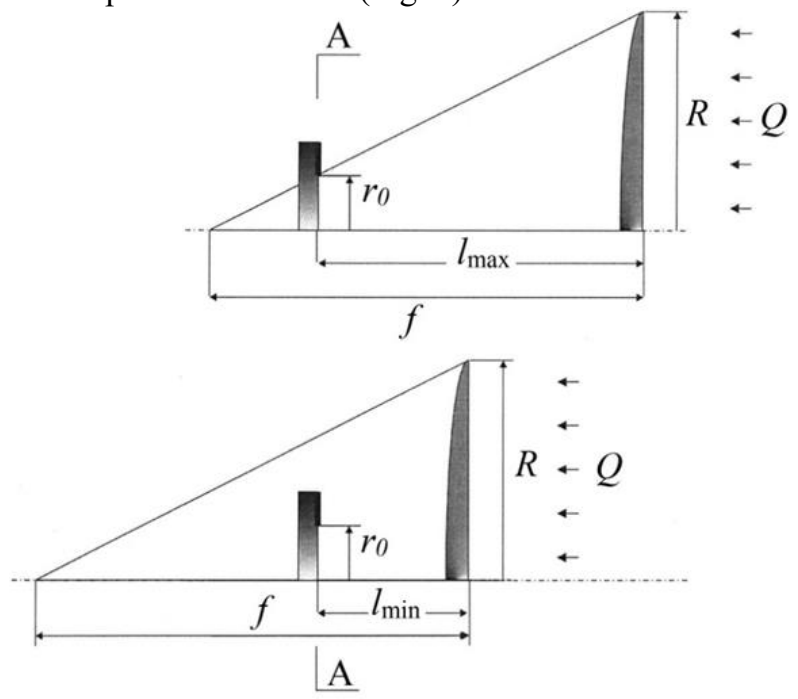

Fig. 1. Geometrical parameters of optical system.

At the time of irradiation heat flow at the opening of the shutter, the lens is moved relative to the sample. Radius of opening of diaphragm is determined by formula

$$
r_{0}=\frac{R}{f}\left(f-l_{\text {мах }}\right),
$$

Variable heat flow density acting on the sample over time $t=0 \div t_{\mathrm{k}}$, calculated by the formula

$$
q(t)=\frac{Q}{\pi}\left\{\frac{f}{R\left[f-\left(l_{\text {мin }}+u t\right)\right]}\right\}^{2}
$$

when moving lens from sample, or on formula

$$
q(t)=\frac{Q}{\pi}\left\{\frac{f}{R\left[f-\left(l_{\mathrm{Max}}-u t\right)\right]}\right\}^{2}
$$


when moving lens towards sample.

Values $l_{\max }, l_{\min }, f$ choose according to inequality

$$
l_{\text {min }}<l_{\text {Max }}<f .
$$

here $R, f$ - the radius and focal distance of lens; $l_{\max }, l_{\min }$ - the maximum and minimum distances between lens and surface of sample; $Q$ - the radiant heat flux arriving on lens; $u, t_{\mathrm{k}}-$ the speed and end time of movement of lens.

Figure 2 shows the results of measurement of density of radiant heat flux depending on distance from lens $l$ to sample surface CS. Three duplicate experiments were carried out to improve the accuracy of experimental data. The design values of $q(l)$ are given on fig. 2 . Calculated values is in good agreement with the experimental data (discrepancy of results doesn't exceed $5 \div 7 \%$ )

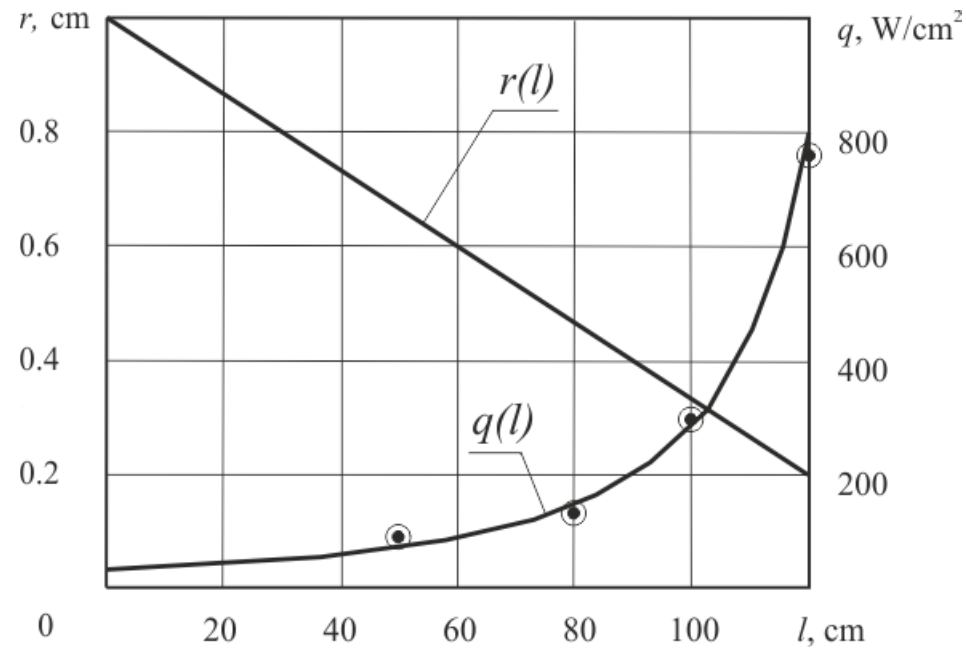

Fig. 2. Dependence of heat flux density on distance.

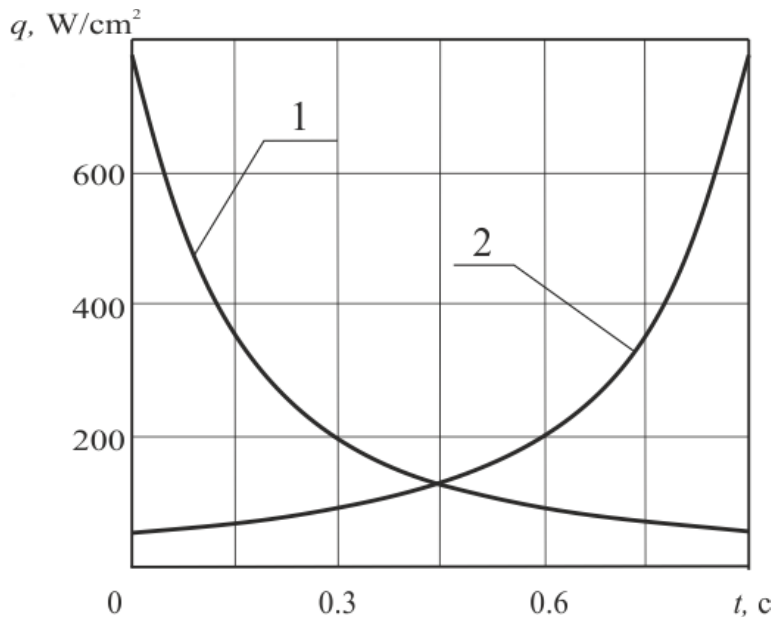

Fig. 3. Dependences of heat flux density on time.

Dependences of heat flux density on time for these conditions, are given in Fig. 3 for two options: reduction of distance from a lens to sample (1) and increase of distance from a lens to sample (2). 


\section{Conclusions}

Thus the offered technique of research of characteristics of ignition of CS allows to expand conditions of radiation of sample:

- the technique allows to define of ignition delay time of samples of CS both at decreasing and at increasing density of radiant heat flux on sample surface.

- changing the speed of movement of the lens along the axis. This allows to vary the dynamic characteristics of the heat flow.

- at variation of geometrical characteristics of optical system (radius and focal distance of lens, diaphragm opening radius) it is possible to take measurements of characteristics of ignition of samples of CS with the broad range of values of the defining parameters.

This research carried out in 2015 was supported by "The Tomsk State University Academic D.I. Mendeleev Fund Program” grant № 8.2.46.2015.

\section{References}

1. V.A. Arkhipov, A.G. Korotkikh, V.T. Kuznetsov, E.S. Sinogina, J. Chem phys. 58, 92 (2007)

2. L.T. De Luca, L. Galfetti, F. Severini, P. Taiariol, V.A. Babuk, B.N. Kondrikov, A.B. Vorozhtsov, G. Klyakin, Progress Combust. Deton. 151, 384 (2004)

3. A.G. Merzhanov, V.V. Barzykin, Some problems of propellant ignition (Preprint of the Inst. of Chem. Phys., Moscow, 1970)

4. L.K. Gusachenko, V.E. Zarko, A.D. Richkov, J. Combust. Explos. Shock 48, 73 (2012)

5. R.Sh. Enaleev, V.A. Mateosov, K.I. Sinaev, B.D. Dinovetskiy, R.Sh. Gainutdinov, Cheboks., Chuvash. State University Publ. 80 (1973)

6. V.A. Arkhipov, V.E. Zarko, A.G. Korotkikh, V.T. Kuznetsov, A.A. Razdobreev, Patent Rus. № 2569641 (2015) 